\title{
Reflectance-Based Imaging Spectrometer Error Budget Field Practicum at the Railroad Valley Test Site, Nevada
}

C alibration and validation determine the quality and integrity of the data provided by sensors and have enormous downstream impacts on the accuracy and reliability of the products generated by these sensors. With the imminent launch of the next generation of spaceborne imaging spectroscopy sensors, the IEEE Geoscience and Remote Sensing Society's (GRSS's) Geoscience Spaceborne Imaging Spectroscopy Technical Committee (GSIS TC) initiated a calibration and validation initiative.

This article reports on a recent reflectance-based imaging spectrometer error budget field practicum focused on radiometric calibration of spaceborne imaging spectroscopy sensors. The field exercise, conducted at Railroad Valley in Nevada, provided valuable training for personnel in a variety of Earth observation (EO) areas, from engineers developing future sensors to calibration scientists actively working in the field.

Future work in this area will focus on analyzing the data acquired as part of the training to answer numerous scientific questions, e.g., understanding the spatial and spectral homogeneity of the site being measured, identifying the optimal sampling to characterize the site, and optimizating the sampling techniques, including looking into the automation of some measurement protocol aspects.

The training exercise was recorded to ensure that the knowledge can be disseminated across the GRSS and wider imaging spectroscopy community.

\section{IMPORTANCE OF CALIBRATION AND VALIDATION}

Calibration is the process of quantitatively defining a system's responses to known, controlled signal inputs, and validation is the process of assessing, by independent means, the quality of the data products derived from those system outputs [1]. As is the case with other EO sensors, the calibration and validation of spaceborne imaging spectroscopy sensors provide fundamental underpinning activities. They play a crucial role in assuring those whose work relies on data provided by spaceborne imaging spectroscopy sensors that the measurements are sound and trustworthy.

It is planned that at least five imaging spectroscopy satellites will be launched over the next five years, with the two most advanced scheduled for liftoff in the next two years [2]. The launch of these sensors requires that suitable, standardized, and harmonized calibration and validation strategies be established to ensure high data quality and comparability between sensor systems. Such activities are extremely important for the community of imaging spectroscopy users. Therefore, the GSIS TC initiated a calibration and validation initiative and has held workshops and training sessions in conjunction with the International Geoscience and Remote Sensing Symposium (IGARSS) in recent years.

As part of the calibration and validation initiative, the GSIS TC conducted a reflectance-based imaging spectrometer error budget field practicum following the IGARSS in Fort Worth, Texas, in July 2017. The purpose and objectives of the training focused on radiometric calibration and, more specifically, on the practical aspects of calculating reflectance-based imaging spectroscopy error budgets. The training encompassed the complete chain of the process, from the laboratory to field measurements.

Six attendees participated in the training, which included a tour of the Remote Sensing Group's laboratory facilities at the University of Arizona, Tucson, and 
a field deployment at the Railroad Valley Playa test site in Nevada. The field training activity included demonstrations of measurement protocols for surface and atmospheric parameters that minimize uncertainties and the collection of data as part of the reflectance-based calibration for an onorbit sensor. An emphasis was placed on techniques and protocols suitable for calibrating imaging spectrometers.

\section{VICARIOUS CALIBRATION}

Vicarious calibration is a crucial component of EO missions, especially for those instruments with limited onboard tuning capabilities. Vicarious calibration refers to techniques that make use of natural or artificial sites on

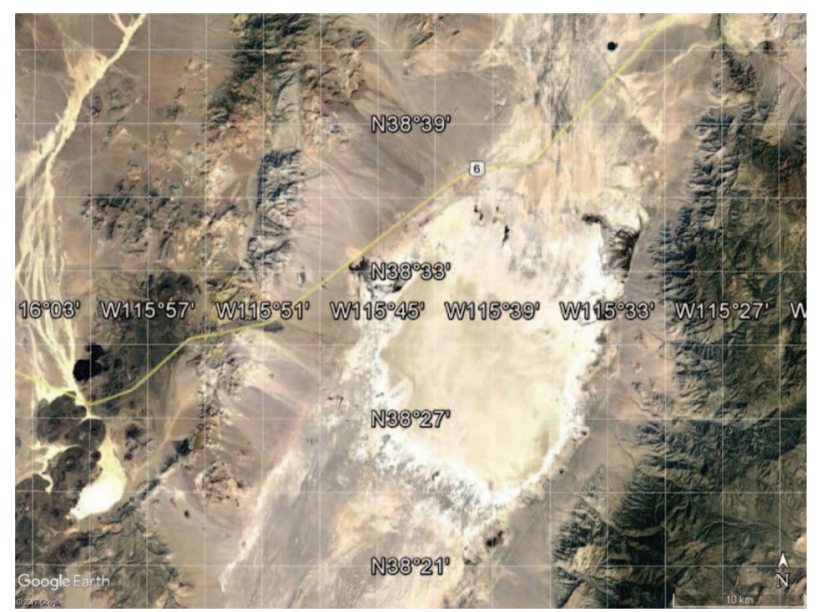

FIGURE 1. An image of the Railroad Valley vicarious calibration site. (Image courtesy of Google Earth.)

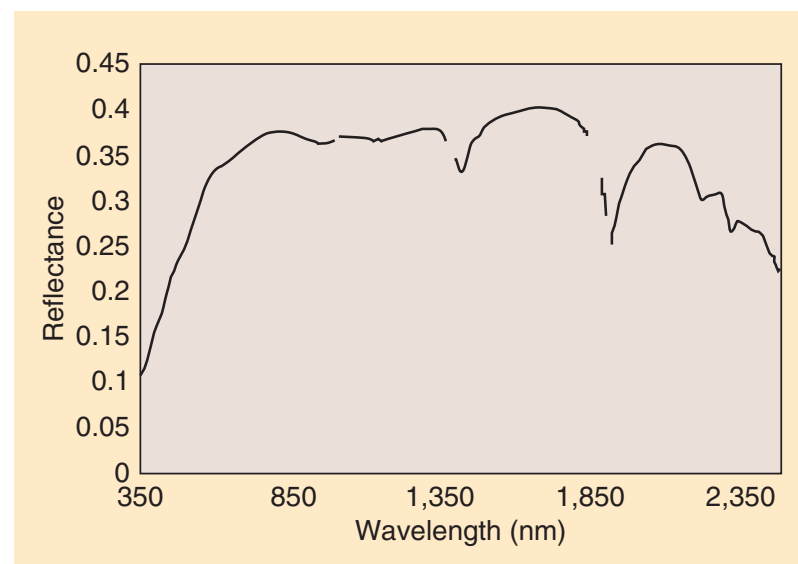

FIGURE 2. The spectral reflectance characteristics of Railroad Valley Playa measured during the training. The regions of water vapor absorption at around 1,400 and 1,900 nm have been removed for clarity. Previous measurements of the Railroad Valley site indicate that the average reflectance is generally greater than $30 \%$ and that the surface is nearly Lambertian at $\pm 20^{\circ}$. Historical data indicate that the reflectance value can be up to $20 \%$ lower in winter. Additionally, seasonal changes in reflectance in response to fluctuations in groundwater levels have been observed. the surface of the Earth for postlaunch adjustment of sensors. The sites used for vicarious calibration are surveyed in near-coincident fashion by the device to be calibrated and by one or more well-tuned and traceable sensors that collect equivalent measurements. These reference sensors are usually ground instruments but can also include spaceborne and airborne equipment.

When selecting a location to be used as a vicarious calibration site, the recommended criteria to be considered were provided by [4]. These include the following characteristics:

D spatial-homogeneous and large

spectral-flat

D temporal-Lambertian, invariant, and stable through time

Dhysical-high altitude and arid.

Also to be considered for the site are logistical constraints, such as access and distance to travel to collect measurements.

A number of vicarious calibration sites have been established in the last few decades, and some have been used on a long-term basis, with permanent instrumentation established as part of the site. One such location is at the Railroad Valley Playa [3], [4], which was established by the University of Arizona, Tucson.

\section{CHARACTERISTICS OF THE RAILROAD VALLEY VICARIOUS CALIBRATION SITE}

The Railroad Valley vicarious calibration site is a dry lake bed playa located in the Nevada desert, approximately $480 \mathrm{~km}$ north of Las Vegas, Nevada, and $160 \mathrm{~km}$ east of Tonopah, Nevada (Figure 1). The size is approximately $15 \mathrm{~km} \times 15 \mathrm{~km}$, and the playa altitude is about $1,435 \mathrm{~m}$ above sea level. The coordinates of the playa are approximately $38^{\circ} 30^{\prime} 14.4^{\prime \prime} \mathrm{N}$ and $115^{\circ} 41^{\prime} 31.2^{\prime \prime} \mathrm{W}$.

The average temperature recorded in July is $19.6{ }^{\circ} \mathrm{C}$, with the highest being $30.6{ }^{\circ} \mathrm{C}$; the average January temperature is $-4.1^{\circ} \mathrm{C}$, with the highest being $4.3{ }^{\circ} \mathrm{C}$, recorded at the closest climate station at Ely, Nevada. [5]. In winter, the cloud cover increases, and periodic snow and water cover can occur. The typical atmospheric conditions at the site include an average aerosol optical depth at $550 \mathrm{~nm}$ of lower than 0.050 [6]. The surface of the dry lake is devoid of vegetation and consists of compacted, clay-rich lacustrine deposits. Figure 2 shows an average spectrum of the site, measured during the training exercise. An interpretation of the spectral features in the short-wave infrared (SWIR) band indicates that the area consists predominantly of montmorillonite and hectorite, and the visible and near infrared shows minor amounts of iron oxides.

\section{INSTRUMENTATION INSTALLED AT RAILROAD VALLEY}

A suite of instruments called the Radiometric Calibration Test Site (RadCaTS) [7] is permanently installed at the Railroad Valley vicarious calibration location. As part of the Radiometric Calibration Network (RadCalNet, www.radcalnet .org), RadCaTS provides surface reflectance data to NASA 
in 10-nm intervals every $30 \mathrm{~min}$ from 9 a.m. to 3 p.m. local standard time. RadCaTS actually produces hyperspectral surface reflectance data at 1-nm intervals (400-2,500 nm), and the surface reflectance is measured every $2 \mathrm{~min}$. Specifically, RadCATS consists of four multispectral eight-channel ground-viewing radiometers (GVRs) measuring across the visible to the SWIR band. These acquire measurements every $10 \mathrm{~min}$, and each GVR footprint is a circle with a diameter of $270 \mathrm{~mm}$ (see Figure 3). These GVRs were comprehensively characterized in the laboratory at the University of Arizona before deployment. Additionally, a transfer radiometer-the Calibration Test Site, Système International-Traceable Transfer Radiometer [8] - is currently being developed to verify the calibration of RadCATS.

Additional instrumentation has been installed to provide information about the atmospheric composition and climate. This includes a CIMEL sun photometer (Figure 3), which is part of the Aerosol Robotic Network (https://aeronet.gsfc .nasa.gov/). This instrument is calibrated at the NASA Goddard Space Flight Center and acquires solar irradiance measurements (direct and sky) in eight channels to characterize the atmospheric composition.

\section{TEST SITE CONDITIONS}

Besides careful campaign planning for the measurements, experiments, and equipment, an essential aspect that should not be neglected during field campaigns is the environmental conditions. This is especially true for desertlike sites such as Railroad Valley, where the conditions may potentially pose occupational, health, and safety risks. Temperatures can reach levels of $40{ }^{\circ} \mathrm{C}$ in the summer, and, because there is no vegetation, operators are exposed to direct sunlight with no shade. The importance of this risk was emphasized as part of the training.

To mitigate the risk from heat and sun, personal equipment such as wide-brim hats, collared shirts with long sleeves, long pants, and sunscreen is required. Sufficient drinking water and something to create shade (e.g., cars and canvas covers) are further requirements that must be considered. Additionally, each field team participant was advised to look out for other participants and pay careful attention for any signs of unusual behavior that might suggest incipient problems and so catch them at an early stage.

\section{CALIBRATION FACILITIES}

\section{AT THE UNIVERSITY OF ARIZONA}

The quality of the data acquired in the field relies significantly on the quality of the reference instruments used. The radiometric characteristics of these instruments must be well understood so that the data can be employed in a traceable manner. To emphasize this crucial component of vicarious calibration, a tour of the calibration facility at the University of Arizona's Remote Sensing Group was included in the training. The tour focused on the facilities used in the calibration and characterization of the reflectance- based measurements and on the traceability of the instruments used in the measurements.

The laboratory calibration facility consists of two adjacent rooms. The first is the control room, where the lamp source and electronics used to control the lamp illuminating the reflectance panels are located. The second is a dark room containing instrumentation for performing radiance calibrations of field and laboratory radiometers as well as calibration of the reference panels. The method includes the use of an FEL lamp calibrated by the National Institute of Standards and Technology and aligned at a clearly defined distance; the light is then reflected off a known reflectance standard viewed by the radiometer at an angle of $45^{\circ}$. The radiance viewed by the radiometer is computed using the calibration of the lamp. An irradiance calibration can also be similarly performed with the radiometer viewing the lamp directly. The technique also includes employing an integrating sphere as the radiance standard.

The laboratory additionally contains instrumentation for the calibration of reference panels. This includes a goniometer (Figure 4) to which the panels are mounted and that is used to orient these panels relative to the lamp as a means of characterizing their bidirectional reflectance characteristics. Other equipment in the laboratory includes a double-pass monochromator used for linearity, signal-to-noise ratio, spectral response, and absolute radiometric calibration of field and laboratory radiometers.

\section{FIELD MEASUREMENTS}

The field training activity emphasized the collection of data as part of the reflectance-based calibration of an on-orbit sensor. For this purpose, several measurement experiments

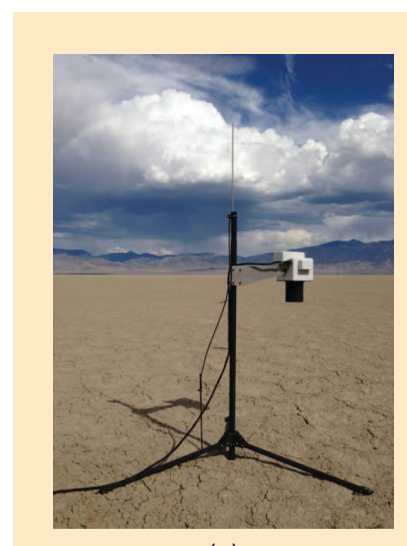

(a)

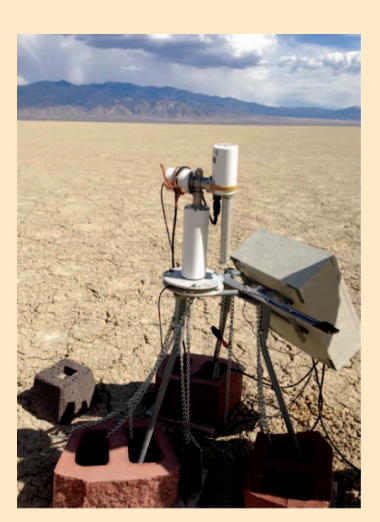

(b)
FIGURE 3. (a) A GVR at Railroad Valley. (b) A CIMEL sun photometer. 


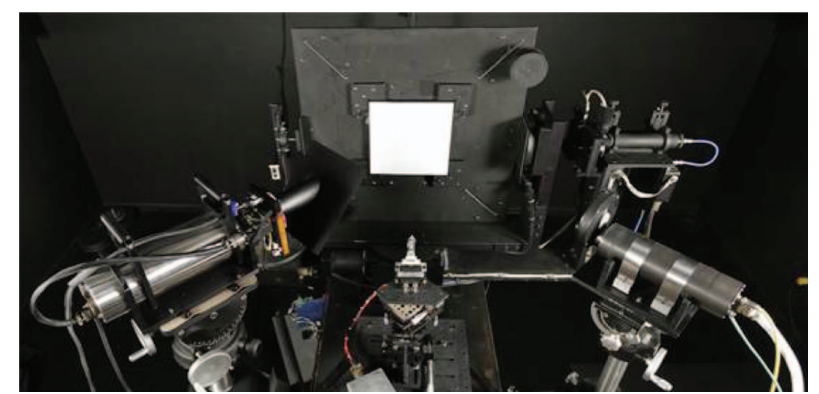

FIGURE 4. A goniometer used to characterize the bidirectional reflectance characteristics of the reference panels. The facility is at the laboratories of the Remote Sensing Group of the University of Arizona. (Photo courtesy of https://wp.optics.arizona.edu/rsg/).

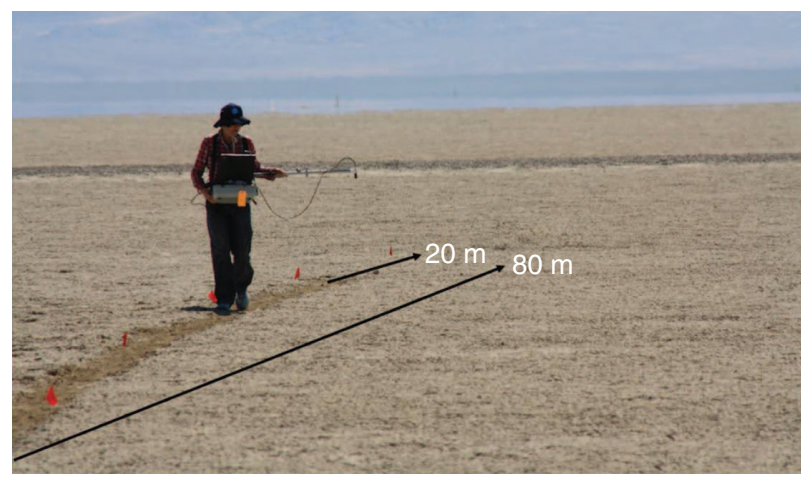

FIGURE 5. The reflectance intercomparison measurements along an $80-\mathrm{m}$ transect. (Photo courtesy of A. Mueller.)

were designed and executed over three consecutive days. The goals of these measurement exercises were

D to allow participants to collect field spectrometer data at the test site as part of an intercomparison exercise for evaluating the repeatability and accuracy of reflectance retrievals

D to test two methods for test site sampling (continuous versus stop-and-stare measurement)

D to perform a reflectance-based calibration of an on-orbit sensor (the field acquisition was scheduled concurrently with the overpass of a Sentinel-2A sensor).

The first intercomparison
A RECORDING OF THE

TRAINING EXERCISE

WAS MADE AND WILL BE

PROVIDED TO THE BROADER

IEEE GRSS AND GENERAL

IMAGING SPECTROSCOPY

COMMUNITIES. exercise was designed to repeat past round-robin experiments across multiple users and days to compare the retrieved reflectance over a relatively short linear transect of about 80 m (Figure 5). Measurements were acquired multiple times on days 1 and 2 at the test site. The intercomparison allowed participants to operate the Analytical System Device field spectrometer multiple times over the site and also provided measurement support (panel setup and documentation) as a way to understand proper protocols and methodologies as well

\section{Continuous Collection}

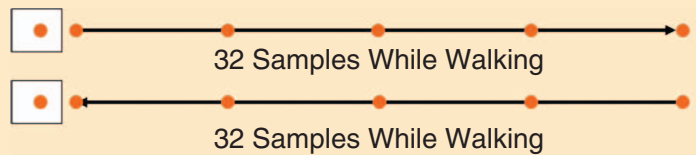

Stop-and-Stare Collection

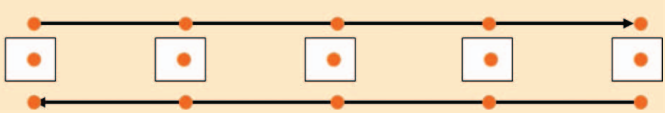

Eight Samples per Location with Operator Standing in Place

FIGURE 6. A schematic representation of the second experiment. Each red dot marks the start of a field spectrometer measurement. A red dot in a rectangle illustrates the spectral measurement of a reference panel.

as the importance of recording critical metadata associated with the acquisition.

The second experiment used the same short linear path (Figure 6) to test the differences between a continuouscollection philosophy across the transect versus a stop-andstare approach. The third exercise allowed participants to put their training into practice by applying the techniques from the first two days to a real-world vicarious calibration campaign during a Sentinel-2A multispectral instrument overflight. The sensor imaged the Railroad Valley site on day 3 of the campaign (Figure 7). The size of the area that was spectrally characterized is suitable for calibrating remote-sensing systems having spatial resolutions of 20-30 m. As they were trained to do during the first and second days, participants used the two approaches to test the comparability of the measurements.

\section{DISCUSSION AND FUTURE PROSPECTS}

The field training exercise conducted at Railroad Valley provided valuable training for sensor engineers, calibration experts, and other scientists working across many EO areas. As part of the training, a comprehensive set of valuable data was acquired that will be helpful in answering a number of questions, including those involving

D the spatial and spectral homogeneity of the site being measured

D the optimal sampling to characterize the site

D the optimization of the sampling methods, including possibly automating some aspects of the measurement procedures.

The data acquired as part of the training will now be analyzed. The trainees have been offered the opportunity to explore additional scientific questions that may be answered with the data, undertake the analysis, and publish the results in an IEEE journal. It is envisioned that the data may be used as partial training material for future GSIS TC courses within the calibration and validation initiatives. A recording of the training exercise was made 


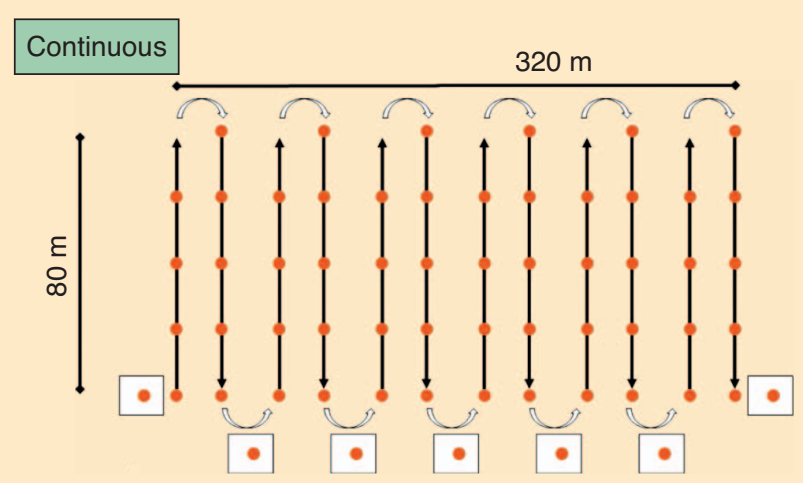

(a)

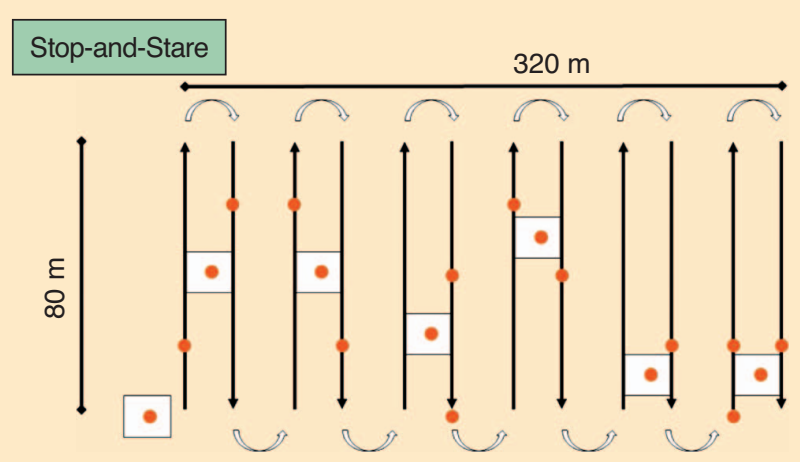

(b)

FIGURE 7. The spectral measurements suitable to perform a reflectance-based calibration of the Sentinel-2A sensor using (a) the continuous collection approach and (b) the stop-and-stare collection approach. Each red dot marks the start of a field spectrometer measurement. A red dot in a rectangle illustrates the spectral measurement of a reference panel.

and will be provided to the broader IEEE GRSS and general imaging spectroscopy communities.

Another calibration and validation training session was conducted prior to IGARSS 2018 in July in Valencia, Spain. For more information on future training sessions, please visit https://igarss2018.org/SummerSchool.asp.

\section{ACKNOWLEDGMENT}

This training exercise was funded by the IEEE Geoscience and Remote Sensing Society as part of the Geoscience Spaceborne Imaging Spectroscopy Technical Committee calibration and validation initiative. We gratefully acknowledge this support.

\section{AUTHOR INFORMATION}

Cindy Ong (cindy.ong@csiro.au) is with the Commonwealth Scientific Industrial Research Organisation, Perth, Australia. She is the chair of the IEEE Geoscience and Remote Sensing Society's Geoscience Spaceborne Imaging Spectroscopy Technical Committee. She is a Member of the IEEE.

Kurt Thome (kurtis.thome@nasa.gov) is with the NASA Goddard Space Flight Center, Maryland. He is a Member of the IEEE.

Uta Heiden (uta.heiden@dlr.de) is with the German Remote Sensing Data Center, German Aerospace Center, Oberpfaffenhofen, Germany. She is cochair of the IEEE Geoscience and Remote Sensing Society's Geoscience Spaceborne Imaging Spectroscopy Technical Committee. She is a Member of the IEEE.

Jeff Czapla-Myers (jscm@optics.arizona.edu) is with the University of Arizona, Tucson. He is a Member of the IEEE.

Andreas Mueller (andreas.mueller@dlr.de) is with the German Remote Sensing Data Center, German Aerospace Center, Oberpfaffenhofen, Germany. He is cochair of the
IEEE Geoscience and Remote Sensing Society's Geoscience Spaceborne Imaging Spectroscopy Technical Committee. He is a Member of the IEEE.

\section{REFERENCES}

[1] CEOS: Committee on Earth Observation Satellites. WGCV. [Online]. Available: http://ceos.org/ourwork/workinggroups/wgcv/

[2] A. Mueller, U. Heiden, and C. Ong, "Current developments of spaceborne imaging spectroscopy missions: Overview, potential cross-links and synergies," in Proc. IEEE Int. Geoscience and Remote Sensing Symp., Milan, Italy, 2015.

[3] K. P. Scott, K. J. Thome, and M. R. Brownlee, "Evaluation of Railroad Valley Playa for use in vicarious calibration," in Proc. SPIE Conf., Denver, CO, 1996, pp. 158-166.

[4] K. J. Thome, "Absolute radiometric calibration of Landsat 7 ETM+ using the reflectance-based method," Remote Sens. Env., vol. 78, no. 1-2, pp. 27-38, 2001.

[5] Yr. (2018, Mar. 30). Weather statistics for Railroad Valley, Nevada (United States). [Online]. Available: https://www.yr.no/place/ United_States/Nevada/Railroad_Valley/statistics.html

[6] K. J. Thome, S. F. Biggar, and W. Wisniewski, "Cross comparison of EO-1 sensors and other Earth resources sensors to Landsat-7 ETM+ using Railroad Valley Playa," IEEE Trans. Geosci. Remote Sens., vol. 41, no. 6, pp. 1180-1188, 2003.

[7] J. S. Czapla-Myers, K. J. Thome, and N. P. Leisso, "Radiometric calibration of Earth-observing sensors using an automated test site at Railroad Valley, Nevada," Canadian J. Remote Sens., vol. 36, no. 5, pp. 474-487, 2010.

[8] S. Biggar, N. Anderson, J. McCorkel, and J. Czapla-Myers, "Earthobserving satellite intercomparison using the Radiometric Calibration Test Site at Railroad Valley," J. Appl. Remote Sens., vol. 12, no. 1, 2017. doi: 10.1117/1.JRS.12.012004. 